Voix et Images

voixetimages

\title{
L’Espace-temps télévisuel et les censures
}

\section{Jean-Pierre Lefebvre}

Volume 2, numéro 1, septembre 1976

Fernand Leduc

URI : https://id.erudit.org/iderudit/200029ar

DOI : https://doi.org/10.7202/200029ar

Aller au sommaire du numéro

Éditeur(s)

Les Presses de l'Université du Québec

\section{ISSN}

0318-9201 (imprimé)

1705-933X (numérique)

Découvrir la revue

Citer cet article

Lefebvre, J.-P. (1976). L'Espace-temps télévisuel et les censures. Voix et Images, 2(1), 130-136. https://doi.org/10.7202/200029ar d'utilisation que vous pouvez consulter en ligne.

https://apropos.erudit.org/fr/usagers/politique-dutilisation/ 


\section{L'Espace-temps télévisuel et les censures}

Dans un article précédent ${ }^{1}$ nous suggérions: 1. que les Québécois sont les plus grands consommateurs de télévision au monde; 2 . que «nous ne regardons pas la télévision, que c'est elle qui nous regarde, c'est elle qui nous consomme", affirmant de la sorte la passivité quasi absolue du téléspectateur; 3 . que la télévision est entre les mains d'hommes d'argent et d'État, l'argent appuyant son pouvoir sur celui de l'État, l'État appuyant le sien sur celui de l'argent, et que par conséquent tout est une affaire de "rentabilité». Afin d'aller plus loin dans la même ligne de pensée, afin de chercher à savoir «de quelle manière " la télévision nous regarde et de quel type est "son" regard, j'aimerais maintenant parler de deux facteurs majeurs dont le premier, l'espace-temps télévisuel, est trop souvent laissé pour compte, et le second, la censure directe ou indirecte, est malheureusement ramené dans la plupart des cas à sa manifestation la plus voyante: l'interdit.

\section{L'ESPACE-TEMPS TÉLÉVISUEL}

Il convient tout d'abord de situer l'appareil de télévision dans l'espace ambiant. On constate que, petit et mobile, il est de prime abord sécurisant, un peu à la façon d'un jouet, d'autant plus que pour quelques dollars de plus on peut le contrôler à distance. On constate en même temps qu'il est le témoin des gestes les plus intimes, dans la cuisine, la salle à manger, la toilette, la chambre à coucher.

Sécurisant de taille et de forme, il l'est encore bien davantage par la grandeur relative de son écran dont le propre est de tout rapetisser, y compris ce que le cinéma avait démesurément agrandi. Le téléspectateur ne se sent jamais physiquement subjugué par ce regard lumineux qui correspond à environ vingt pour cent du regard humain tandis que le cinéma peut atteindre à mille pour cent.

L'espace télévisuel est toutefois doublement miniaturisé, car il n'est pas autonome: il fait partie, contrairement à l'espace cinématographique, de l'espace familier des hommes. Son intensité, son existence même sont ainsi limitées à l'extrême parce qu'elles sont perpétuellement menaçées par les niveaux d'occupation ou de saturation des sens autres que la vue, principalement l'ouie et l'odorat - et parfois même la vue elle-même.

De manière conséquente, pour pallier à la précarité de l'espace télévisuel, on crée de plus en plus un temps télévisuel artificiel et disproportionné. Artificiel, tout d'abord, parce que toute insertion d'un corps étranger, telle la publicité, à l'intérieur d'un sujet spécifique, en brise, en 
trahit fatalement le rythme concret et abstrait. Disproportionné, ensuite, parce qu'en plus de l'accélération du rythme extrinsèque au sujet, on en force le rythme intrinsèque au niveau de la forme aussi bien que du fond.

L'accélération de la forme intrinsèque au sujet est de la plus simple évidence; elle se traduit par la surabondance de mouvements de lentilles (je dis bien de lentilles et non de caméra pour făíre une distinction très nette entre un zoom-in-zoom-out et un travelling arrière et avant: dans un travelling c'est le spectateur lui-même qui s'approche ou s'éloigne, dans un zoom c'est le contraire, c'est le sujet-objet qui s'approche ou s'éloigne du spectateur); elle se traduit par ailleurs dans le jeu des comédiens et la mise en scène elle-même en laissant croire, comme dans les romanssavons, que la rapidité est signe d'efficacité.

Plus subtile est l'accélération du fond. Mais si on analyse toute matière dramatique créée et produite spécifiquement pour la télévision, on se rend vite compte qu'on est en présence d'une "dramaturgie du dénouement", rien de moins rien de plus. Peu importe la manière dont les choses vont se terminer, comment les héros vont s'en sortir, et peu importe le pourquoi et surtout le comment, à moins qu'il ne s'agisse d'un comment purement technique et/ou technologique (cf. «Mission impossible ") qui devient alors le sujet lui-même.

Bien entendu cette dramaturgie du dénouement est en très grande partie rendue possible autant que nécessaire par l'accélération du rythme extrinsèque et intrinsèque de la forme; car sans le bris du fond, sans les interruptions de la publicité, le dénouement viendrait trop vite ${ }^{2}$.

Bref, si l'espace télévisuel est à peu près inexistant, le temps télévisuel a pour fonction de passer et de faire passer. Mais on va encore plus loin malgré l'accélération fulgurante du rythme concret et abstrait du sujet télévisuel: on s'assure que la piste sonore est parfaitement saturée pour permettre, même à distance, même le nez dans les chaudrons, de suivre l'action ou l'histoire; on appuie à coup sûr le dénouement par une musique équivalente qui nous indique que c'est le temps de lever le nez des chaudrons afin de recueillir la récompense du message final qui, soit dit en passant, est toujours individuellement et socialement apaisant, réconfortant. On peut donc prétendre que la formule populaire est juste: nous écoutons la télévision beaucoup plus que nous ne la regardons.

Ne reste plus aux hommes d'État et d'argent que le loisir et le bénéfice d'utiliser au maximum les avantages et les décifiences d'un procédé de communication où cette dernière, autre avantage, ne se réalise toutefois qu'à sens unique, que de haut en bas, jamais de bas en haut. Les hommes d'argent et d'État peuvent ainsi tout orienter, tout contrôler. En quelque sorte tout censurer. 


\section{LA ET LES CENSURES}

Officiellement, on prétendra qu'il n'y a pas de censure dans une démocratie comme la nôtre, que la liberté d'expression est un fait accompli de même que la liberté de choix. Mais quelle est la liberté d'expression du peuple à la télévision! Et quelle autre liberté de choix a-t-il que celle de fermer l'appareil!

C'est vrai, il n'y a pas de censure à la télévision: il y en a plusieurs, et des plus raffinées, pour la plupart issues des normes de productionconsommation.

\section{La censure légale}

Qu'on le veuille ou non, il y a d'abord une censure directe relevant du code criminel pour une part (avouée) et des intérêts politiques pour une autre part (non avouée).

La première est plus ou moins ambiguë. Elle ne lésine pas en matière de mœurs sexuelles, par exemple, bien qu'elle ne fasse rien contre la violence orgiaque et gratuite d'un nombre incalculable de séries policières et de films du même genre, et bien que le CRTC n'intervienne même pas dans la programmation de ces séries et films aux heures où ce sont essentiellement les enfants qui regardent la télévision.

La censure "politique", pour sa part, a rarement à s'affirmer directement ou publiquement, car elle agit essentiellement à la source, c'està-dire au niveau de la production. Ou alors, si elle n'a pu empêcher la production, elle refusera "l'achat" et ainsi donc empêchera la diffusion.

\section{La censure par classification horaire}

Ne considérons plus maintenant que ce qui a été produit et est par le fait même susceptible d'être diffusé (consommé).

Le premier niveau de censure se fera par classification horaire du ou des sujets. A Radio-Canada on remarque ainsi trois grandes divisions dans la programmation-horaire, l'une pour le «peuple" en général, une autre pour les «intellectuels" et une dernière qui devrait satisfaire les deux: le sport et les informations. (Remarquons tout de suite qu'en Amérique du Nord, seuls le sport et les "conventions" politiques, ou les élections, sont rois et maîtres sur la programmation-horaire de la télévision; on amputera un film comme Godfather s'il le faut mais à tout prix on respectera la symétrie maniaque des horaires, sauf dans le cas du sport ou de la politiqueconsidérée-comme-un-sport-national.) À Télé-métropole, comme sur la plupart des chaînes privées, on ne s'embêtera pas de cette dichotomie et on visera simplement la consommation populaire. 
Cette forme de censure, très souvent inconsciente, se fait essentiellement au nom de la rentabilité (cote d'écoute) et/ou en fonction de l'auditoire-cible visé, ce qui revient à la même chose.

Ce qu'on censure d'abord et avant tout de cette façon c'est la qualité. Ce qu'on détruit avant tout c'est l'idée de qualité à l'intérieur de la culture populaire réduite au dénominateur commun de la consommationdivertissement. Quant à la Culture, ou celle bourgeoisement considérée comme telle, elle est enclavée dans les parenthèses d'une programmationhoraire spéciale (ex.: “les Beaux dimanches") ou plus simplement nocturne (23 heures en montant). Quant à notre culture, elle ne bénéficie des heures de pointe que si elle subit la comparaison avec la culture étrangère, et cela à deux niveaux : premièrement la "qualité " établie habituellement sur le fait de la reconnaissance à l'étranger (c'est logique); deuxièmement celui de la conformité aux normes de rythme et de dramaturgie du dénouement dont nous avons parlé antérieurement. La programmation des films québécois ou canadiens à Radio-Canada, aux “Grands Films » ou sinon la nuit, est caractéristique en ce sens, aussi bien que l'absence quasi totale de ce même cinéma sur les ondes de Télé-métropole.

De toute évidence la programmation-horaire, et la programmation tout court, de toutes les télévisions au Canada, est hautement significative du néo-colonialisme d'intérêt et d'habitude pratiqué par nos hommes d'État et d'argent. Et ceci, presque illogiquement dans un sens, contre la «production» nationale.

Quoi qu'il en soit, les canaux de la censure par programmationhoraire ainsi creusés, il est impossible de les faire dévier: l'achat et la production ne visent qu'à les remplir, qu'à les alimenter de produits conformes. Et la conformité, rappelons-le, est étrangère.

\section{La censure par modification de la classification-horaire}

C'est ainsi que si par un hasard très rare un produit fait conformément aux exigences de la classification-horaire semble tout à coup y échapper, ou par le fond (l'idéologie) ou par la forme (la baisse éventuelle de la cote d'écoute), il s'agit simplement de le faire sortir de l'orbite prévue. Un exemple type: les huit demi-heures réalisées par Gilles Sénécal de Radio-Canada sous le titre de «Qu'en penses-tu, toi?". Destinées à un public jeune, enfants-adolescents, pour d'obscures raisons elles aboutissent en avant-programme, à sept heures et demi, des "Beaux Dimanches", au moment d'ailleurs où ne prévaut que la fièvre olympique et estivale. Point n'est besoin d'épiloguer.

Cette forme de censure n'est en somme que la conséquence de celle de la programmation-horaire. 


\section{La censure par encerclement}

Le système, ne serait-ce que pour afficher le libéralisme qu'il prétend être le sien, en vient toutefois à faire quelques apparentes concessions (il faut malgré tout qu'il soigne sa propagande). Je dis bien apparentes car on est certain, au point de départ, que le produit insolite, de fond ou de forme, sera rejeté de la même façon qu'un merlan orphelin dans un banc de morues par les pêcheurs-consommateurs qui ne peuvent y trouver leur compte.

C'est la similitude des modèles et des moules, pour la plupart étrangers disions-nous, et l'appui tapageur des "gros" commanditaires à ces même modèles qui permettent "d'encercler" le dit produit insolite, de le ramener à la normalité.

On n'insistera jamais assez, en effet, sur la coloration que la publicité des commanditaires ultra-puissants donne aux sujets auxquels ils accordent leur appui et leur confiance; car personne dans notre société d'ultra-consommation n'est porté à en mettre en doute le sérieux, sinon la nécessité, puisqu'ils vendent les produits de base (nourriture, vêtements, essence, etc.), ou les produits de luxe de base (bière, savon, désodorisant, etc.), ou puisqu'ils vendent la possibilité d'acheter ces mêmes produits (compagnies de finance, banques, etc.). Si donc ces mêmes commanditaires, après avoir sanctionné de leurs dollars des émissions prestigieuses, telle "les Grands Films, " semblent (mais ceci malgré eux, simplement par la volonté des programmateurs) accorder leur appui à des films comme Kamouraska, ou Réjeanne Padovani, ou Bingo, dans l'esprit et dans le regard du téléspectateur il y a déjà un certain réconfort, il prévoit à l'avance ne pas être trop dérouté. Et s'il l'est, les extraits du prochain programme vont vite lui faire oublier son ennui, vont vite lui faire absoudre le ou les commanditaires qui, il ne faut pas l'oublier, ont droit eux aussi à certaines erreurs, certaines faiblesses, celles de la libre concurrence. C'est ainsi que la libre concurrence rend tout semblable, sinon semblablement médiocre.

On comprendra l'extrême prudence des programmateurs qui doivent le plus possible éviter de piquer ou de décevoir les commanditaires car les organismes pour lesquels ils travaillent perdraient alors leur double source de revenus, c'est-à-dire les grosses compagnies (pour la plupart des multi-nationales) et leur public; donc eux perdraient leur job.

La censure par encerclement pourrait donc se définir ainsi: l'intégration à la programmation-horaire régulière de pointe d'éléments non orthodoxes qui le deviennent cependant à cause de la puissance du modèle préalable et de celle du commanditaire qui lui est assimilé. 


\section{La censure par surabondance}

On aboutit maintenant à la résultante d'à peu près toutes les formes de censure antérieurement mentionnées: la censure par surabondance, ou par immersion totale, quantitativement parlant.

II s'agit de noyer l'idéologie dans la sur-production et la sur-diffusion (donc de ne promouvoir que l'idéologie de la production et de la consommation).

À cet égard j'ai toujours imaginé avec horreur l'encéphalogramme du téléspectateur québécois moyen (quatre heures et demi de télévision par jour). L'esprit, comme l'estomac, a des niveaux de tolérance vite atteints et au-delà de ce seuil naturel c'est nécessairement l'indigestion externe et interne.

II n'est pas besoin de faire des enquêtes scientifiques pour admettre qu'il est impossible d'assumer, de digérer, de comprendre, d'analyser et de ressentir vraiment le flot des réalités de tous ordres transmises par un flot également puissant d'impulsions sonores et visuelles qui constituent, réalités et impulsions, le quotidien télévisuel. Devant un tel flot c'est forcément la démission. Ou le règne de l'indifférence, qui est le contraire de la conscience et de la responsabilité.

Sans entrer dans le détail, on peut constater que les émissions d'information sont les plus grandes victimes de cette surabondance, cela de deux manières. Tout d'abord parce qu'elles sont presque infailliblement en sandwich entre des émissions de divertissement qui ont pour ainsi dire toujours le premier et le dernier mot. Ensuite parce qu'on en est venu, devant cette triste réalité, à concevoir l'information à la manière d'un divertissement ou d'un show (cf. "le 60 "). Quant aux "Dossiers" ils suivent habituellement la pente du divertissement et sont en retard par rapport à la réalité de la dramaturgie du dénouement qui, elle, a depuis longtemps imposé le «mal» de la violence, de la folie, de la pornographie; ces dossiers tombent donc presque immanquablement dans le piège du dualisme le plus archaïque et s'appliquent à vouloir dire que si le «mal» n'est pas un bien c'est parce qu'on fait trop mal le bien.

Indigestion et hémorragie il y a donc jusqu'à la torture, jusqu'au massacre des derniers neurones encore vivants. Ainsi,. mercredi le 7 juillet à Radio-Canada vous pouviez voir, dans la série "C'était hier", et à 11 heures du soir évidemment, «l'Algérie française", bien évidemment entrecoupée d'une bonne douzaine de publicités, ce qui constitue un acte assez épouvantable de barbarie, mais immédiatement suivie de "Doucement, les basses", comédie avec Alain Delon, Paul Meurisse et Nathalie Delon. Comment l'un peut-il ne pas annuler l'autre! Autre exemple: cette année chaque long métrage canadien ou québécois présenté dans la série "Cinéma d'ici", cela à 11 heures et plus, était suivi, quelque part entre une heure et deux heures du matin, d'un long métrage étranger. En se- 
maine. C'était, bien entendu, pour suppléer au mécontentement éventuel du téléspectateur non encore habitué aux indigences ou aux spécificités de son cinéma national; alors rien de mieux que de revenir au "modèle d'habitude » avant le sommeil qui fait tout oublier.

\section{L'auto-censure}

"Ils n'en mourraient pas tous, mais tous étaient frappés. " On comprendra aisément que dans un système basé essentiellement sur la rentabilité de la production et la production de la rentabilité, l'individu, parfaitement isolé et conséquemment amené à croire qu'il est le seul responsable de sa propre richesse ou pauvreté matérielle, on comprendra aisément que cet individu fasse indirectement ou directement le jeu du système. On comprendra qu'il se dise à lui-même que le jour viendra où, sa fidélité reconnue, sinon son talent, par ses "employeurs", ces derniers lui seront reconnaissants et lui permettront une échappée. Mais cet individu oublie que le système tient sa force majeure de sa compartimentation et que chaque compartiment a une porte à deux battants.

L'individu devra donc, dans la plupart des cas, se soumettre ou abandonner. A moins qu'il ne soit promu. Car le système punit indifféremment la fidélité et l'infidélité de la suprême récompense de la promotion. Car plus vous êtes hauts dans l'échelle sociale, mieux on peut vous neutraliser - ou vous faire tomber.

\section{En guise de conclusion}

Ou nous vivons dans un pays qui ne connaît aucune forme de censure, ou nous devons laisser de côté notre conception univoque et pascalienne de la censure et dénoncer les privlèges, les pouvoirs de la technocratie comme autant d'abus et de causes de frustration et de castration.

Ou nous acceptons l'esclavage et la torture mentale du petit jouetrobot, ou nous réinventons la production culturelle à partir des nécessités intérieures et extérieures, humaines et sociales, de l'homme collectif que nous sommes.

Sinon la télévision aura été un échec parfait de communication, donc un instrument de manipulation.

Jean-Pierre Lefebvre

1. Voir Voix et images, vol. I, n³, avril 1976, p. 445-447.

2. Au cas où certains l'ignoreraient encore, toutes les séries américaines sont découpées d'avance avec ces avis: "Place commercial here." 